\title{
Social control of reproduction in breeding and non-breeding male naked mole-rats (Heterocephalus glaber)
}

\author{
C. G. Faulkes and D. H. Abbott \\ MRC/AFRC Comparative Physiology Research Group, Institute of Zoology, Zoological Society of \\ London, Regent's Park, London NWI 4RY, UK
}

\begin{abstract}
Summary. Eight male naked mole-rats, from three colonies were studied in captivity. When non-breeding male naked mole-rats were removed from their colonies and paired with a non-breeding female, or removed and housed singly for 6 weeks before pairing with a female, concentrations of urinary testosterone and plasma luteinizing hormone $(\mathrm{LH})$ increased significantly $(P<0.05)$. Concentrations of these hormones were highest while the males were singly housed: urinary testosterone (mean \pm s.e.m.) increased from $8.2 \pm 1.3 \mathrm{ng} / \mathrm{mg}$ urinary creatinine $(\mathrm{Cr})$ in a non-breeder in a colony to $49 \cdot 1 \pm 5 \cdot 5 \mathrm{ng} / \mathrm{mg} \mathrm{Cr}$ when singly housed and $21 \cdot 8 \pm 2.5 \mathrm{ng} / \mathrm{mg} \mathrm{Cr}$ when paired with a female. Plasma LH concentrations increased from $4.7 \pm 1.0 \mathrm{miu} / \mathrm{ml}$ when a nonbreeder in a colony to $19.8 \pm 4.0 \mathrm{miu} / \mathrm{ml}$ when singly housed and $9.9 \pm 1.1 \mathrm{miu} / \mathrm{ml}$ when paired with a female. After pairing with a female, the pattern of urinary testosterone secretion in the male was synchronized with the ovarian cycle of the female mate, such that urinary testosterone concentrations were significantly higher during the early follicular phase of the female's cycle $(P<0.05)$.

These results suggest that active suppression of reproductive physiology by social cues occurs in non-breeding male naked mole-rats, and that this is readily reversible if social cues are removed and males are housed singly. When a male was subsequently paired with a female, endocrine suppression was partially reimposed on the reproductively active males, such that urinary testosterone concentrations were suppressed to values similar to those in non-breeding males, except for periods prior to mating. The breeding female may therefore control the reproductive physiology of both breeding and non-breeding males in colonies of naked mole-rats.
\end{abstract}

Keywords: reproductive suppression; naked mole-rats; hystricomorph rodent; testosterone; luteinizing hormone

\section{Introduction}

The naked mole-rat, Heterocephalus glaber, is a highly social hystricomorph rodent inhabiting the arid regions of East Africa, including Kenya, Ethiopia and Somalia. Living entirely underground in colonies which often contain $40-90$, but sometimes up to 300 , individuals, their burrow systems contain communal nest and toilet chambers and an extensive network of foraging tunnels, which may total 2-3 km in length (Jarvis, 1981, 1985; Brett, 1986, 1991). A characteristic and unique feature of naked mole-rat colonies is the extreme behavioural and reproductive division of labour, similar to that of eusocial insects, whereby reproduction is restricted to a single breeding female, the 'queen' and 1-3 males; this division is seen in both captive (Jarvis, 1981; Lacey \& Sherman, 1991; Faulkes et al., 1991a) and wild colonies (Jarvis, 1985; Brett, 1991). The remaining colony members do not breed and, in females, ovulation is blocked (Faulkes et al., 1990a), possibly as a result of 
inadequate plasma luteinizing hormone $(\mathrm{LH})$ concentrations arising from impaired hypothalamic gonadotrophin-releasing hormone (GnRH) secretion (Faulkes et al., 1990b).

Among male naked mole-rats, there are clear behavioural and physiological differences between breeders and non-breeders. Only breeding males are solicited by, and mate with, the queen (Jarvis, 1991; Lacey \& Sherman, 1991). Breeding males have higher concentrations of plasma LH, greater LH responses to administration of exogenous $\mathrm{GnRH}$, and higher concentrations of urinary testosterone than non-breeders (Faulkes et al., 1991b). These observations suggest that reproduction is suppressed in non-breeding male naked mole-rats, but concentrations of reproductive hormones are sufficient to support spermatogenesis, and these males apparently produce mature spermatozoa (Faulkes et al., 1991b; Jarvis, 1991).

To study further the endocrine differences between breeding and non-breeding male naked mole-rats, and to investigate the physiological changes that occur when non-breeding males are removed from the suppressing influences of their colonies, eight males from three captive colonies were studied. Concentrations of plasma LH and urinary testosterone were determined in these individuals while they were non-breeders in a colony, when removed and housed singly for a minimum of 6 weeks and when paired with a female.

\section{Materials and Methods}

\section{Experimental procedures}

Animal housing. Captive colonies of naked mole-rats were maintained at the Institute of Zoology, London, using artificial Perspex burrow systems containing nest, food and toilet chambers (described by Faulkes et al., 1990a, b). Animals were numbered and identified by a system of toe clipping and tattoos. Altogether eight males and eight females from three captive colonies were used in this study.

Reproductive activation in non-breeding males. To investigate the hormonal changes that occur when males become reproductively active for the first time, non-breeding males were removed from their colonies and either paired directly with a non-breeding female from the same colony $(n=3)$, or removed, housed singly for a minimum of 6 weeks and then paired with a female of approximately the same age and from the same colony, who had also been housed singly for 6 weeks $(n=5)$ and had therefore become reproductively active (see Faulkes et al., 1990a). The males were all nonbreeding adults whose body mass ranged from 19 to $51 \mathrm{~g}$, aged 5-40 months at the time of separation (the mean body mass for captive animals is $\sim 30 \mathrm{~g}$ ). While reproductive status was the most important factor in choosing the males for the experiment, animals of a range of ages and body sizes were picked to investigate whether this influenced the subsequent endocrine changes. Animals were housed singly before pairing to ascertain whether the physical presence of a mate was required to stimulate reproductive activation. After pairing, casual observations of mating were recorded.

Urine sampling was carried out 1-4 days a week between 08:00 and 18:00 h, from 240 days before, until 330 days after, separation. A total of 226 samples collected from the eight males was subjected to testosterone radioimmunoassay. To quantify the time taken for urinary testosterone concentrations to rise after separation to values comparable to those of breeding males, a urinary testosterone value that reflected breeding status had to be chosen. The criterion used was the mean testosterone value for breeding males, previously calculated as $24 \mathrm{ng} / \mathrm{mg}$ urinary creatinine $(\mathrm{Cr})$ (142 samples from nine breeding males; Faulkes et al., 1991b). The latency from separation to a rise in urinary testosterone concentrations to concentrations comparable to those in breeders was defined as the time in days from separation (Day 0 ) to the day on which urinary testosterone concentrations rose from sustained low values to reach $24 \mathrm{ng} / \mathrm{mg} \mathrm{Cr}$ or more. The latency values were obtained by extrapolating from this value on individual testosterone profiles plotted against time.

A total of 6-8 plasma samples was collected from each male at intervals of 2-4 weeks, up to 80 days before separation, during the singly housed period, and for up to 50 days after pairing with a female. LH determinations were carried out on plasma, rather than urine, because the latter was not suitable for the bioassay method employed. Bioactive LH was measured in preference to immunoreactive LH, because of the greater sensitivity of the method and because the former only detects biologically active forms of $\mathrm{LH}$.

Monitoring of ovarian cyclicity in females. To investigate whether concentrations of reproductive hormones in male naked mole-rats were correlated with the reproductive status of their female mate, urinary testosterone concentrations in the males were plotted relative to the ovarian cycle of the female. The ovarian cycles of females were followed by measurement of urinary progesterone by radioimmunoassay (Faulkes et al., 1990a). This involved plotting the urinary progesterone profiles obtained from females against time as follows: Day 0 was designated as the day before a sustained rise in urinary progesterone above $2 \cdot 0 \mathrm{ng} / \mathrm{mg} \mathrm{Cr}$ and was assumed to correspond approximately to the day of ovulation. Day I was designated as the start of the luteal phase; the end of the luteal phase was taken as the 
day on which a rapid and sustained fall in urinary progesterone below $2.0 \mathrm{ng} / \mathrm{mg} \mathrm{Cr}$ occurred. Urinary testosterone concentrations in males were plotted against the respective female cycle, assuming a mean total cycle length of 34 days ( 6 days follicular, 28 days luteal: Faulkes et al., 1990a).

\section{Sampling}

Urine. Urine was chosen for routine steroid hormone determination in males and females in captive colonies in preference to blood because collection is non-invasive and disturbance to the animals is therefore minimized (Faulkes et al., 1990a, 1991b). Urine has been widely used as a medium for hormonal analysis in other species (e.g. Lasley, 1985; Hodges, 1986).

Urine sampling involved the removal of all the shavings from the toilet chamber in each colony and wiping the chamber clean with tissue paper. Immediately after each urination, the sample was collected in a glass pipette and the toilet chamber was wiped clean with tissue paper. Samples were placed in a freezer within $1 \mathrm{~h}$ of collection, and stored at $-20^{\circ} \mathrm{C}$ until hormone determination.

Blood. Animals were hand-held, the tip of the tail was cut with a sterile scalpel blade and blood $(\sim 200 \mu$ l) was collected by capillary action using heparinized microhaematocrit tubes. Blood samples were collected within $2-4 \mathrm{~min}$ of animal capture from our captive colonies, and afterwards the wound was treated with an antibiotic powder (Aureomycin) and the animal returned to its colony. The samples were stored on ice for a maximum of $2 \mathrm{~h}$ before being centrifuged for $5 \mathrm{~min}$ at $500 \mathrm{~g}$, and the plasma was stored at $-20^{\circ} \mathrm{C}$ before LH determination.

\section{Hormone determinations}

Radioimmunoassay of testosterone. Before testosterone assay, all urine samples were subjected to a determination of creatinine as described by Bonney et al. (1982). All urinary testosterone concentrations were expressed as mass per milligram of creatinine $(\mathrm{mg} / \mathrm{Cr})$ to correct for dilution of urine.

Testosterone concentrations were determined in urine $(50-100 \mu)$, extracted with diethyl ether by radioimmunoassay (RIA) following celite chromatography, using sheep antitestosterone antibody no. 505 (MRC Reproductive Physiology Unit, Edinburgh, UK; see Hodges et al., 1987), as previously described and validated for the naked mole-rat (Faulkes et al., 199lb).

The sensitivity of the assay (determined as $90 \%$ binding) was $2.0 \mathrm{pg} /$ tube. At an average dilution of urine, this was equivalent to $<1.0 \mathrm{ng} / \mathrm{mg} \mathrm{Cr}$. Interassay precision, expressed as the coefficient of variation for repeated determinations of a quality control $(2.22 \mathrm{ng} / \mathrm{mg} \mathrm{Cr})$, was $15.1 \%(n=4)$ for quality controls subjected to column chromatography and $16.9 \%(n=4)$ for quality controls extracted only. Intra-assay variation was $7.5 \%(n=7)$.

Progesterone radioimmunoassay. Progesterone in female urine was measured in samples $(50-100 \mu 1)$, extracted with petroleum ether, by RIA without chromatography, as previously described and validated for the naked mole-rat (Faulkes, 1990; Faulkes et al., 1990a, 1991a).

The sensitivity limit of the assay (determined as $90 \%$ binding) was $10 \mathrm{pg} /$ tube. Intra- and interassay precision, expressed as the coefficient of variation for repeated determinations of a quality control, was $6 \cdot 0(n=11)$ and $7 \cdot 1 \%$ $(n=19)$, respectively.

Luteinizing hormone bioassay. LH was measured using an in-vitro bioassay based on the production of testosterone by dispersed mouse Leydig cells (Van Damme et al., 1974). The method and validation for the naked mole-rat have been described previously (Faulkes, 1990; Faulkes et al., 1990a, b). Plasma samples were assayed in duplicate at two dilutions of $1: 10$ and $1: 20$, or 1:20 and 1:40, as a routine check for parallelism, and compared with a rat LH standard (the rLH antigen preparation: rLH-I-7) over the range $2-0.0625$ milli-international units (miu)/ml. The testosterone produced was measured by RIA.

The sensitivity of the assay (determined at $90 \%$ binding) was $0 \cdot 1$ miu per tube. Intra- and interassay precision for the whole assay, expressed as the mean coefficients of variation for repeated determinations of an LH quality control $(1.53 \mathrm{miu} / \mathrm{ml})$, were $10(n=15)$ and $16 \%(n=9)$, respectively.

\section{Statistical analysis}

Urinary testosterone and plasma $\mathrm{LH}$ data were analysed using one-way analysis of variance for repeated measures following log transformation of data. Log transformation of plasma LH concentrations was carried out as a standard procedure, to increase the linearity of the data and to reduce the heterogeneity of variance (Sokal \& Rohlf, 1981). Results quoted in the text are means \pm s.e.m for the non-transformed data; the figures reflect the data as the antilog of the means transformed for statistical analysis, with their $95 \%$ confidence limits. Comparisons of individual transformed means were made post hoc using Duncan's multiple-range test with a level of significance of $P=0.05$ (Helwig \& Council, 1979). 


\section{Results}

Plasma LH concentrations rose significantly from $4 \cdot 7 \pm 1.0$ to $19 \cdot 8 \pm 4.0 \mathrm{miu} / \mathrm{ml}$ when males were removed from their colonies and housed singly $(\mathrm{F}(2,15)=4.88 ; P<0.05)$. When paired with a female, plasma LH concentrations were less than when housed singly, but greater than when the males were of non-breeding status $(9 \cdot 9 \pm 1 \cdot 1 \mathrm{miu} / \mathrm{ml}$; Fig. 1(a)).
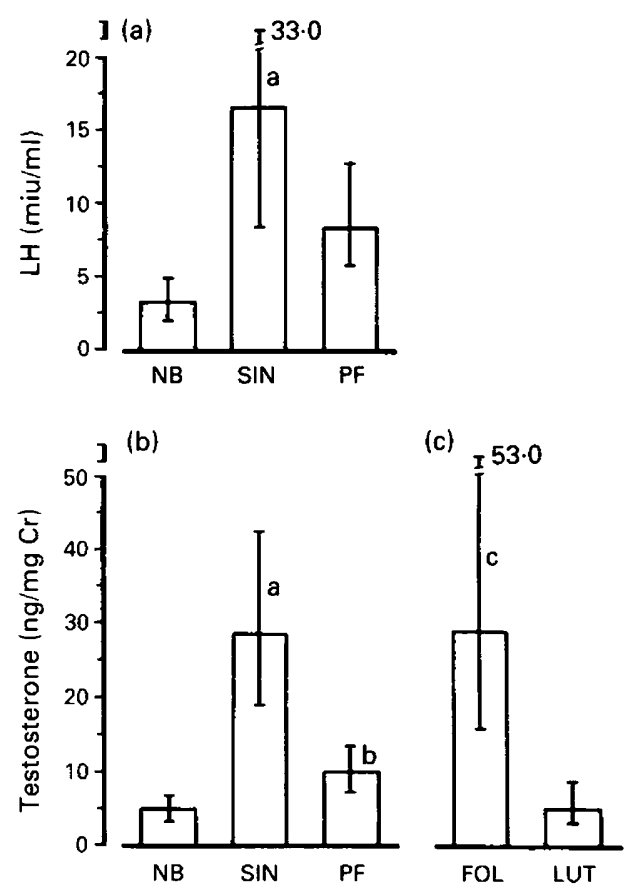

Fig. 1. Concentrations of (a) plasma luteinizing hormone ( $\mathrm{LH}$ ) and (b) urinary testosterone, in eight male naked mole-rats when non-breeders in a colony (NB) were removed from their colonies and housed singly for a minimum of 6 weeks (SIN), then paired with a female $(n=5)$, or removed and paired directly with a female ( $n=3$; PF); (c) concentrations of urinary testosterone in males after pairing, during the follicular phase \pm 3 days (FOL), and during the luteal phase (LUT) of the female mate's ovarian cycle. Data are expressed as the antilog of the transformed means, with $95 \%$ confidence limits. (a) $P<0.05$ vs. NB and PF; (b) $P<0.05$ vs. NB; (c) $P<0.05$ vs. LUT and NB. Duncan's multiple-range test following one-way ANOva for repeated measures.

Similar trends were observed in urinary testosterone concentrations, which rose significantly from $8.2 \pm 1.3 \mathrm{ng} / \mathrm{mg} \mathrm{Cr}$ (mean \pm s.e.m.) to reach a maximum while males were housed singly $(49 \cdot 1 \pm 5 \cdot 5 \mathrm{ng} / \mathrm{mg} \mathrm{Cr})$. In animals paired with a female, urinary testosterone concentrations were lower than in those singly housed at $21.8 \pm 2.5 \mathrm{ng} / \mathrm{mg} \mathrm{Cr}$, but significantly higher than in males of non-breeding status $(\mathrm{F}(2,18)=16 \cdot 1 ; P<0.001$; Fig. 1(b)). Concentrations of urinary testosterone were significantly higher during the female's follicular phase ( \pm 3 days), at $47.8 \pm 6.2 \mathrm{ng} / \mathrm{mg} \mathrm{Cr}$ (mean \pm s.e.m.), than during the luteal phase of the female's cycle $(8.9 \pm 1.6 \mathrm{ng} / \mathrm{mg} \mathrm{Cr}$ ), and when a non-breeder in a colony $(\mathrm{F}(3,19)=17.9 ; P<0.001)$. There were insufficient plasma samples for a comparison of $\mathrm{LH}$ concentrations in males collected during the follicular and luteal phases of the female.

From examination of individual urinary testosterone profiles (Fig. 2a, b) the mean time between separation and a rise in urinary testosterone to $24 \mathrm{ng} / \mathrm{mg} \mathrm{Cr}$, the mean for breeding males, was 
calculated as $5 \cdot 3 \pm 1 \cdot 0$ days ( $n=6$ males: five housed singly, one paired directly with a female). The age and body mass at which the males were removed from their parent colonies did not appear to influence the latency of the rise in urinary testosterone concentrations, which ranged from 3 to 9 days, while the ages ranged from 5 to 40 months, and body masses from 19 to $51 \mathrm{~g}$.

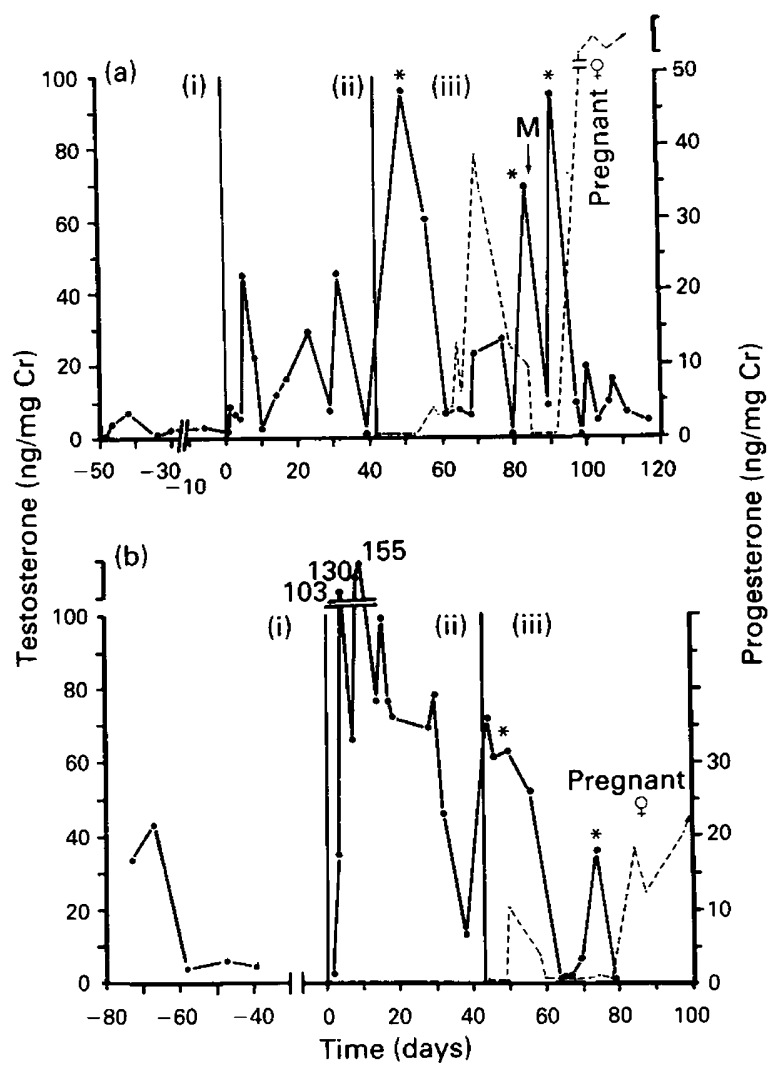

Fig. 2. Urinary testosterone profile for naked mole-rats: (a) male 1, aged 15 months, body mass $30 \mathrm{~g}$, and (b) male 8 , aged 15 months, body mass $32 \mathrm{~g}$, (i) when a non-breeder in a colony, (ii) when removed and housed singly for 6 weeks and (iii) when paired with a female mate who had also been housed singly for 6 weeks; $(--)$ ovarian cycles of the females, the urinary progesterone profile; $\mathrm{M}$, observation of mating; *, a peak in urinary testosterone $>24 \mathrm{ng} / \mathrm{mg} \mathrm{Cr}$, which coincides with the follicular phase of the female mate's cycle.

When animals were housed singly, urinary testosterone concentrations were maintained at higher values than when the males had non-breeding status and, in some instances, reached $>100 \mathrm{ng} / \mathrm{mg} \mathrm{Cr}$ (Fig. 2(b)). After pairing with a female, urinary testosterone concentrations still reached high values, but these episodes were mainly confined to the follicular phase of the female's cycle. For example, male 1 had three peaks in urinary testosterone after pairing with female 4 (Fig. 2(a)), all of which occurred when urinary progesterone in the female was undetectable or $<2.0 \mathrm{ng} / \mathrm{mg} \mathrm{Cr}$, indicating the follicular phase of the ovarian cycle (Faulkes et al., 1990a). During the second follicular phase of female 4 at about Day 90 , two peaks in urinary testosterone were recorded, and mating occurred. Conception occurred during this time and the female became pregnant (Fig. 2(a)). Similarly, male 8 showed two peaks in urinary testosterone after pairing with female 10 , both of which coincided with the follicular phase of the female's cycle, although the first peak also carried on into the luteal phase (Fig. 2(b)). Again, successful fertilization occurred during the second ovarian cycle. Male 8 (Fig. 2(b)) had an isolated peak in urinary testosterone 
$>24 \mathrm{ng} / \mathrm{mg} \mathrm{Cr}$ between Days -65 and -75 , while a non-breeder in its parent colony. The cause of this is unknown, because during this time the queen was between Days 21 and 40 of pregnancy; the possible effect of a period of oestrus in the breeding female was therefore not implicated. Nonbreeding males have occasionally been shown to have urinary testosterone concentrations that overlap with the lower range of those of breeding males (Faulkes et al., 1991b).

Of 15 peaks of urinary testosterone ( $>24 \mathrm{ng} / \mathrm{mg} \mathrm{Cr}$ ) that were observed after pairing, 11 corresponded to periods when the female mate was apparently in the follicular phase of her cycle. By combining the urinary testosterone data from the individual profiles, and comparing the values with the respective female mate's cycle, a composite urinary testosterone profile over the female ovarian cycle was constructed (Fig. 3).

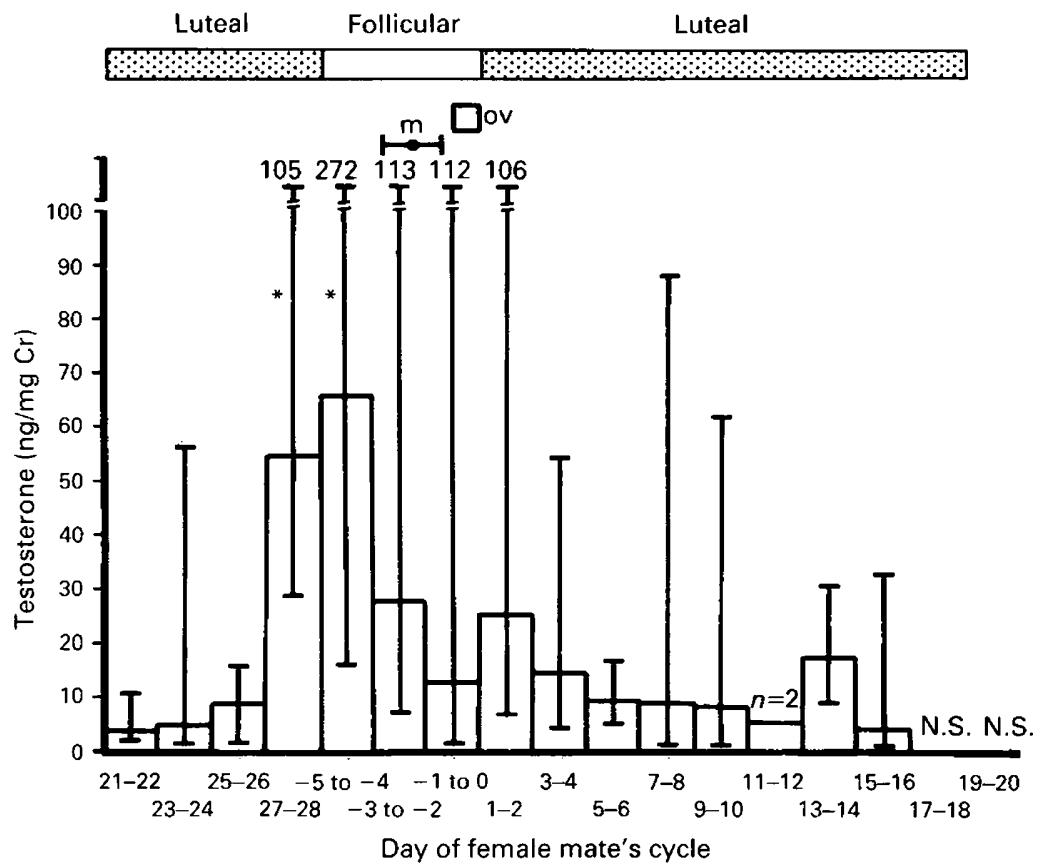

Fig. 3. Concentrations of urinary testosterone in 68 samples collected from five male naked mole-rats over 12 female ovarian cycles. Data are represented as the antilog of the means transformed for statistical analysis, with $95 \%$ confidence limits, and are plotted relative to the ovarian cycle of the female mate, in 2-day intervals. A mean total cycle length of 34 days was assumed. M, mean \pm s.e.m. day of mating, $n=6$ observations; N.S., no samples collected from this period; ov, presumed day of ovulation; *, $P<0.05$ vs. Days $15-16,19-20$ and 21-22. Duncan's multiple-range test following one-way ANOVA for repeated measures.

Urinary testosterone concentrations in males paired with females reached significantly higher concentrations on Days 27-28, at the end of the luteal phase, and on Days -5 to -4 , the first part of the follicular phase of the female cycle (mean \pm s.e.m.: $63.0 \pm 13 \cdot 1$ and $73.2 \pm 21.9 \mathrm{ng} / \mathrm{mg} \mathrm{Cr}$, respectively), compared with Days 15-16, 21-22 and 23-24 of the luteal phase $(7 \cdot 2 \pm 3 \cdot 4,5 \cdot 7 \pm 2 \cdot 2$ and $10 \cdot 5 \pm 6 \cdot 1 \mathrm{ng} / \mathrm{mg} \mathrm{Cr}$, respectively; $\mathrm{F}(14,36)=2 \cdot 03 ; P<0 \cdot 05 ;$ Fig. 3$)$.

\section{Discussion}

Like their female counterparts, non-breeding male naked mole-rats have clear endocrine differences from breeders. In non-breeding males, these differences are characterized by low concentrations of plasma LH and urinary testosterone. However, unlike their female counterparts, the 
endocrine deficiencies in non-breeding males did not reflect a suppression of mature gamete development, as spermatogenesis was observed in all males (Faulkes et al., 1991b). In non-breeding females, ovulation was suppressed and most ovarian follicular development was arrested at a preantral stage (Faulkes, 1990; Faulkes et al., 1990). The endocrine differences amongst males were readily reversible when non-breeding males were removed from the social factors that caused reproductive suppression in their colonies. When the males were paired with a reproductively active female mate, successful conceptions occurred, in some cases after only one ovarian cycle, at the second period of the female's oestrus.

The rapidity with which reproductive activation occurred in non-breeding males was comparable to that observed in non-breeding females, where urinary progesterone concentrations reached values indicative of a luteal phase $\sim 8$ days after removal from their colonies (Faulkes et al., 1990a). The age and body mass of the non-breeding males before separation did not appear to influence the latency of onset of reproductive activation, with small, young males giving similar results to those of larger, older individuals. Therefore, like females (Faulkes, 1990; Faulkes et al., 1990a), males were able to undergo puberty and attain breeding status well before they had reached the maximum body size of $60-70 \mathrm{~g}$.

When non-breeding males were removed from their colonies and housed singly, both urinary testosterone and plasma LH concentrations increased markedly to reach high values that were, for the most part, sustained over the period of isolation. Whilst in this singly housed condition, the males were released from reproductive suppression and were able to undergo increases in reproductive hormones without the physical presence of a female. However, when the males were subsequently paired with a reproductively active female, a degree of suppression or control over both $\mathrm{LH}$ and testosterone secretion was reintroduced by the female. This reproductive control resulted in episodes of high urinary testosterone in the male that were synchronized with the ovarian cycle of the female, such that peak concentrations of testosterone in the male occurred during the late luteal and early follicular phase of the female's cycle. Although plasma LH concentrations were not studied in the same detail as urinary testosterone, the former showed the same overall trends as the latter over the three housing conditions (see Fig. 1(a)). Therefore, it is assumed that the changes in urinary testosterone reflect similar changes in plasma $\mathbf{L H}$ and plasma testosterone. These results imply that, in males, active suppression of pituitary and testis secretion of LH and testosterone, respectively, occurs during periods outside the late luteal to early follicular phase of the breeding female's cycle. Release from suppression, and possibly stimulation of the male by the female, would appear to coincide with periods just prior to the presumed time of oestrus in the female.

The physiological function of an increase in circulating testosterone in the male prior to oestrus in his female mate may be to facilitate sexual behaviour in the male. The role of testosterone in promoting male sexual behaviour is well documented in other species, for example, in male rats (for review see Feder, 1978). Whether the increases in testosterone may also stimulate spermatogenesis or affect sperm viability is unknown. It is possible that the increases in testosterone may alter or increase secretions from the accessory sex organs, thereby enhancing the survival and viability of spermatozoa in the ejaculate. The stimulatory effect of androgens on the accessory sex organs of other species is well known (for review see Coffey, 1988).

The periodic peaks in urinary testosterone concentrations seen in these males when paired with females could be a response to changes in the behaviour of either or both sexes or to a stimulatory pheromonal cue given off by the female. There is circumstantial evidence that both of these factors may be involved. High frequencies of mutual anogenital nuzzling have been observed between breeding male and female naked mole-rats (Jarvis, 1991; Lacey \& Sherman, 1991), suggesting that the male is continuously exposed to any chemosignals derived from the genitalia and reproductive tract of the female. In some other rodent species, vaginal secretions of oestrous females contain pheromones, which may be critical for the stimulation of normal male sexual behaviour. For example, an anosmic male golden hamster will not mate with an oestrous female even though she 
may advertise her receptive state in other ways (Singer et al., 1980). A striking example of how a female may stimulate the reproductive endocrinology of a male can be found in mice. In this species, unfamiliar females are able to induce an increase in plasma testosterone in the male after 30-60 min (Macrides et al., 1975), while exposure to the urine of females was sufficient to produce significant increases in LH in the male recipient (Maruniak \& Bronson, 1976).

There is strong evidence to suggest that direct behavioural contact with the queen, rather than a primer pheromone contained in the urine of the queen, plays the major role in suppression of reproduction in both non-breeding male and female naked mole-rats. Daily transfer of soiled bedding and litter between parent colonies and separated singly housed animals, or male-female pairs, neither delayed nor prevented the onset of their reproductive activation (Faulkes, 1990). Daily bedding and litter transfer, coupled with the rotation of groups of non-breeding animals between the parent colony and singly housed females every 2 days, did not delay or prevent the separated females from becoming reproductively active (Faulkes et al., 1991; T. E. Smith unpublished data).

The results from this study of singly housed and paired males suggest that not only does the queen suppress reproduction in non-breeding females, but that she may also control the reproductive endocrinology of the breeding male(s).

The queen selects her breeding partners from the males in the colony and also initiates mating behaviour (Jarvis, 1991). It is interesting to speculate that such strict controls over reproductive physiology by the queen may also extend to the non-breeding males in the colony: in one captive colony in which the queen died, concentrations of urinary testosterone in all the non-breeding males of the colony increased to values comparable to those when males are singly housed, indicating a release from suppression (C. G. Faulkes, unpublished data). Such control of reproduction of males and females in a social group by a single dominant individual is apparently without precedent among mammals.

We thank the NIDDKD, Baltimore, MD, USA and the National Hormone and Pituitary Program at the University of Maryland School of Medicine for the rat LH preparation (rLH-I-7) and GnRH; M. J. Llovett and the laboratory animal staff at the Institute of Zoology for care and maintenance of the animals, Professor A. P. F. Flint and Dr H. D. M. Moore for criticism of the manuscript, and T. Dennett and M. J. Walton for preparation of the figures. This work was supported by an MRC/AFRC Programme Grant, a project grant from the Wellcome Trust (to D. H. Abbott) and an SERC Research Studentship (to C. G. Faulkes).

\section{References}

Bonney, R.C., Wood, D.J. \& Kleiman, D. G. (1982) Endocrine correlates of behavioural oestrus in the female giant panda (Ailuropoda melanoleuca) and associated hormonal changes in the male. J. Reprod. Fert. 64, 209-215.

Brett, R.A. (1986) Ecology and behaviour of the naked mole-rat Heterocephalus glaber (Ruppell) Rodentia: Bathyergidae. Ph.D. Thesis, University of London.

Brett, R.A. (1991) The ecology of naked mole-rat colonies: burrowing, food, and limiting factors. In The Biology of the Naked Mole-Rat, pp. 137-184. Eds P. W. Sherman, J. U. M. Jarvis \& R. D. Alexander. Princeton University Press, New York.

Coffey, D.S. (1988) Androgen action and the sex accessory tissues. In The Physiology of Reproduction, pp. 10811119. Eds E. Knobil\& J. Neill. Raven Press, New York.

Faulkes, C.G. (1990) Social suppression of reproduction in the naked male-rat, Heterocephalus glaber. Ph.D. Thesis, University of London.
Faulkes, C.G., Abbott, D.H. \& Jarvis, J.U.M. (1990a) Social suppression of ovarian cyclicity in captive and wild colonies of naked mole-rats, Heterocephalus glaber. J. Reprod. Fert. 88, 559-568.

Faulkes, C.G., Abbott, D.H., Jarvis, J.U.M. \& Sherrifi, F. (1990b) LH responses of female naked mole-rats, Heterocephalus glaber, to single and multiple doses of exogenous GnRH. J. Reprod. Fert. 89, 317-323.

Faulkes, C.G., Abbott, D.H., Liddell, C.E., George, L.M. \& Jarvis, J.U.M. (1991a) Hormonal and behavioural aspects of reproductive suppression in female naked mole-rats Heterocephalus glaber. In The Biology of The Naked Mole-Rat, pp. 426-445. Eds P. W. Sherman, J. U. M. Jarvis \& R. D. Alexander. Princeton University Press, New York.

Faulkes, C.G., Abbott, D.H. \& Jarvis, J.U.M. (1991b) Social suppression of reproduction in male naked mole-rats, Heterocephalus glaber. J. Reprod. Fert. 91, 593-604. 
Faulkes, C.G., Abbott, D.H. \& Smith, T.E. (1991) Socially induced suppression of reproduction in colonies of naked mole-rats. Appl. Behav. Sci. 31, 293-294.

Feder, H.H. (1978) Specificity of steroid hormone activation of sexual behaviour in rodents. In Biological Determinants of Sexual Behaviour, pp. 395-424. Ed. J. B. Hutchison. Wiley, Chichester.

Helwig, J.T. \& Council, K.A. (1979) SAS Users guide. SAS Institute Inc. Cary, North Carolina.

Hodges, J.K. (1986) Monitoring changes in reproductive status. Int. Zoo Yb. 24/25, 126-130.

Hodges, J.K., Cottingham, P., Summers, P.M. \& Yingnan, L. (1987) Controlled ovulation in the marmoset monkey (Callithrix jacchus) with human chorionic gonadotrophin following prostaglandin induced luteal regression. Fert. Steril. 48, 229-305.

Jarvis, J.U.M. (1981) Eu-sociality in a mammalcooperative breeding in naked mole-rat Heterocephalus glaber colonies. Science, $N Y$ 212, 571-573.

Jarvis, J.U.M. (1985) Ecological studies on Heterocephalus glaber, the naked mole-rat, in Kenya. Nat. Geographic Sci. Rep. 20, 429-437.

Jarvis, J.U.M. (1991) Reproduction. In The Biology of The Naked Mole-Rat, pp. 384-425. Eds P. W. Sherman, J. U. M. Jarvis \& R. D. Alexander. Princeton University Press, New York.

Lacey, E.A. \& Sherman, P.W. (1991) Social organization of naked mole-rat (Heterocephalus glaber) colonies: evidence for a division of labour. In The Biology of The Naked Mole-Rat, pp. 275-336. Eds J. U. M. Jarvis, P. W. Sherman \& R. D. Alexander. Princeton University Press, New York.

Lasley, B.L. (1985) Methods for evaluating reproductive function in exotic species. Adv. Vet. Sci comp. Med. 30, 209-228.

Macrides, F., Bartke, A. \& Daltreio, S. (1975) Strange females increase testosterone levels in male mice. Science, NY 189, 1104-1106.

Maruniak, J.A. \& Bronson, F.H. (1976) Gonadotropic responses of male mice to female urine. Endocrinology 99, 963-969.

Singer, A.G., Macrides, F. \& Agosta, W.C. (1980) Chemical studies of hamster reproductive pheromones. In Chemical Signals: Vertebrates and Aquatic Invertebrates, pp. 365-375. Eds D. Müller-Schwarze \& R. M. Silverstein. Plenum Press, New York.

Sokal, R.R. \& Rohlf, F.J. (1981) Biometry: The Principles and Practice of Statistics in Biological Research, 2nd edn. W. H. Freeman and Company, New York.

Van Damme, M.-P., Robertson, D.M. \& Diczfalusy, E. (1974) An improved in vitro bioassay method for measuring luteinizing hormone (LH) activity using mouse Leydig cell preparations. Acta endocr., Copnh. $77,655-671$.

Received 29 October 1990 